\section{$\overline{\substack{\text { hommes } \\ \text { \& migrations }}}$}

\section{Hommes \& migrations}

Revue française de référence sur les dynamiques

migratoires

$1310 \mid 2015$

Fashion Mix

\title{
L'arménien occidental, cent ans de diaspora
}

Entretien avec Anaïd Donabedian, linguiste, responsable des études arméniennes à l'Institut national des langues et civilisations orientales (Inalco), réalisé par Marie Poinsot, rédactrice en chef.

\section{Marie Poinsot}

\section{(2) OpenEdition \\ Journals}

Édition électronique

URL : http://journals.openedition.org/hommesmigrations/3194

DOI : 10.4000/hommesmigrations.3194

ISSN : 2262-3353

\section{Éditeur}

Musée national de l'histoire de l'immigration

\section{Édition imprimée}

Date de publication : 1 avril 2015

Pagination : 171-176

ISBN : 978-2-919040-31-5

ISSN : $1142-852 X$

\section{Référence électronique}

Marie Poinsot, «L'arménien occidental, cent ans de diaspora », Hommes \& migrations [En ligne], 1310 | 2015, mis en ligne le 14 décembre 2015, consulté le 14 septembre 2020. URL : http:// journals.openedition.org/hommesmigrations/3194 


\section{REPÉRAGE}

\section{L'ARMÉNIEN OCCIDENTAL, CENT ANS DE DIASPORA}

Entretien avec ANAÏD DONABEDIAN, linguiste, responsable des études arméniennes à l'Institut national des langues et civilisations orientales (Inalco), réalisé par Marie Poinsot, rédactrice en chef.

Hommes \& Migrations : Plus de 10 millions d'Arméniens se sont dispersés dans le monde. Comment les langues arméniennes de ces diasporas se sont-elles articulées dans ces différents contextes, notamment au Proche-Orient, en Russie, en Europe occidentale, aux États-Unis et en Amérique du Sud?

Anaïd Donabedian : Les foyers de la diaspora ont des profils très différents à plusieurs égards, qui expliquent des différences sensibles dans leur situation linguistique. Plusieurs facteurs interviennent, les plus importants étant le type de construction politique de l'État dans lequel ils sont installés, et notamment le statut des communautés, leur structuration en vagues de migration, leur type d'ancrage géographique dans l'espace urbain.

Les communautés linguistiquement les plus vivantes sont celles du Proche-Orient, où la langue est restée vernaculaire : dans les quartiers à prédominance arménienne de Beyrouth, Alep, Damas, l'arménien est très présent dans l'espace public, que ce soit à l'oral ou à l'écrit (à Bourj Hamoud à Beyrouth, les noms des grandes banques sont transcrits en arménien). La scolarisation dans les écoles communautaires est la norme, même si elle suscite des débats et n'est pas exclusive. De plus, des contraintes nationales pèsent sur le contenu de l'enseignement (en Turquie et en Syrie, les programmes nationaux sont enseignés dans la langue dominante, l'arménien étant officiellement réduit à la dimension religieuse). Au Liban, les Arméniens sont également sensibles au multilinguisme ambiant (l'arabe dans les couches populaires, l'anglais ou le français s'y ajoutant dans les couches moyennes-supérieures). Cependant, avec un réseau de 28 écoles, une presse et une activité éditoriale diversifiées, une vie culturelle en arménien, le Liban qui, avec la guerre, a nourri d'une grande partie de son intelligentsia les communautés de France, du Canada, de Californie, d'Amérique latine, d'Australie, demeure une référence linguistique dans toute la diaspora, le centre normalisateur. En France, où la centralisation linguistique est importante, la pratique de la langue est beaucoup plus fragile, même si les écoles bilingues franco-arméniennes connaissent un succès croissant depuis une quinzaine d'années. L'attachement à la langue reste important, mais, comme l'a montré notre étude le niveau de compétence nécessaire pour satisfaire aux exigences identitaires est minimal, en l'absence de nécessité de communiquer en arménien. Les vagues de migration en provenance du ProcheOrient depuis les années 1970 ont joué un rôle essentiel dans la revitalisation linguistique de la communauté. Le même phénomène peut être observé en Amérique où les communautés issues du Proche-Orient ont constitué de nouveaux terroirs communautaires (comme Little Armenia à 


\section{REPÉRAGE}

Los Angeles). Les comportements linguistiques et sociaux y restent étonnamment proches de ceux du Proche-Orient, alors que les familles issues des premières vagues de migration (1896-1922) ont cédé au modèle intégrationniste des États-Unis qui tolère les communautés à la première génération, pour parvenir à une intégration complète à la troisième génération (bien décrit par le linguiste Joshua Fishman dès les années 1960).

\section{H\&M : Dans quelle mesure les langues armé- niennes des diasporas peuvent-elles maintenir leur vitalité hors d'une territorialité ? Le retour ou la référence à la langue de l'Arménie sont- ils utiles pour préserver cette permanence lin- guistique?}

A. D. : La question est vitale et, à ce titre, le démantèlement de la communauté arménienne de Syrie inquiète beaucoup, de même que les velléités d'émigration de nombreux Libanais, Arméniens inclus, manquant de confiance dans l'avenir politique et économique du pays. Le contact entre l'ar-

L'afflux de migrants venant

d'Arménie est l'un des défis

auxquels se confrontent

les écoles arméniennes dans le monde, la plupart du temps en optant pour une adaptation

de l'enfant à l'arménien

occidental, dont la préservation

reste l'un des objectifs de ce réseau scolaire. ménien occidental et l'arménien oriental, s'il a été limité pendant la période soviétique, n’a jamais été rompu : même au ProcheOrient, nombreux sont ceux qui ont effectué des études supérieures en Arménie, dès l'époque soviétique, les intermariages ne sont pas rares, et il existe une certaine

fluidité entre les deux variantes de cette même langue. Dès qu'il s'agit d'écrit, cependant, on ne peut rester dans l'entre-deux : certains pans de la grammaire sont totalement distincts, l'orthographe réformée aux débuts de l'Arménie soviétique n'est pas acceptée par la diaspora et, enfin, les références littéraires et les effets du contact de langues renvoient à deux univers assez distincts, auxquels les locuteurs tiennent collectivement assez pour que la distinction reste encore forte. L'afflux de migrants venant d'Arménie est l'un des défis auxquels se confrontent les écoles arméniennes dans le monde, la plupart du temps en optant pour une adaptation de l'enfant à l'arménien occidental, dont la préservation reste l'un des objectifs de ce réseau scolaire. Certaines initiatives existent cependant, comme l'atelier Mgnig à Paris, qui concerne des enfants arménophones et, de fait, peut se permettre de viser une coexistence harmonieuse des deux variantes, ou encore certaines écoles de Californie, dont le nombre d'élèves permet de dédoubler certaines classes. De plus en plus d'arménophones accèdent aux deux variantes, même s'ils n'en pratiquent généralement qu'une. Parallèlement, il est intéressant de noter que, même en diaspora, la territorialisation des communautés tend à revenir au goût du jour. En France, par exemple, Alfortville attire actuellement les primo-arrivants et de jeunes couples de troisième ou quatrième génération. Cela pourrait renforcer le tissu communautaire déjà important et avoir un impact sur son profil linguistique.

\section{H\&M : Pouvez-vous nous expliquer la distinc- tion entre arménien occidental et oriental ?}

A. D. : Au XIXe siècle, deux standards littéraires ont émergé : l'arménien occidental, fondé sur les dialectes occidentaux parlés dans l'Empire ottoman, et l'arménien oriental, sur les dialectes orientaux parlés dans le Caucase, désormais russe, et l'Iran. L'arménien classique, écrit depuis le V'e siècle, et qui a donné lieu à une littérature prestigieuse, cesse alors d'être la seule langue d'enseignement et de production littéraire pour devenir peu à peu une référence historique, restée vivante dans le domaine religieux. Ainsi, les Arméniens, avant leur diasporisation, parlaient l'arménien standard occidental ou oriental pour ceux qui avaient été scolarisés ou pour les couches urbaines, des dia- 
lectes arméniens et le turc (certaines régions parlaient d'ailleurs en guise de dialecte une variante locale de turc) ou le russe. Cent ans après, certains dialectes ont survécu, en Arménie, au Nagorno-Karabakh, dans l'Akhalkalak, en Abkhazie, quelques îlots en Turquie, à Kessab en Syrie ou à Anjar au Liban, les générations qui les parlent ne sont pas encore éteintes dans les villes de Syrie et du Liban, mais les deux standards sont la référence de tout locuteur arménien. L'arménien oriental connaît certes des variations (notamment en Iran où la prégnance du dialecte local sur l'écrit reste importante), mais la référence étatique constitue un point de repère stable. II n'en est pas de même pour l'arménien occidental qui est une langue de diaspora pluricentrique, sans autorité politique commune et n'ayant nulle part un statut de langue majoritaire, ce qui la fragilise nécessairement, et explique que l'Unesco l'ait classée langue en danger en 2010.

\section{H\&M : L’arménien est-il davantage perçu comme un patrimoine culturel à conserver ou est-il pratiqué comme une langue vernaculaire familiale?}

A. D. : La langue est considérée par tous les Arméniens comme un des éléments essentiels du patrimoine arménien, et même comme le marqueur identitaire dominant. L'arménien reste vernaculaire dans les communautés dont la structure politico-sociale le permet, a fortiori lorsqu'elles conservent un ancrage territorial, via des quartiers ou des banlieues de prédilection. On peut affirmer qu'il n'existe pas d'Arménien monolingue dans le monde, puisque même dans les campagnes d'Arménie on parle le russe. Le bilinguisme, et même le multilinguisme, est donc la règle, et l'arménien en tant que langue minorée n'est souvent pas dominant. Lorsque l'arménien n'est plus réellement vernaculaire, il reste préservé dans le domaine religieux (le service religieux est pratiqué en arménien classique, et l'homélie, nor- malement en arménien moderne, est aujourd'hui de plus en plus souvent dans la langue locale, ou bilingue), dans certains domaines de la vie (des études ont montré la prégnance de l'arménien en matière de cuisine, de mariage ou de traditions, alors que la langue locale prend le relais pour ce qui concerne la technologie, l'économie, le sexe...), ou encore dans certaines situations, qu'il s'agisse d'un emploi cryptique (pour ne pas être compris au moment d'une négociation, par exemple) ou
Il n'y a aucun moyen aujourd'hui en France de recenser la communauté se reconnaissant d'origine arménienne, les chiffres évoqués et qui tournent autour de 400000 à 500 000, sont des estimations faites à partir de recoupements. empathique (l'inclusion d'éléments d'arménien servant alors à souligner la potentielle complicité). L'ensemble de ces pratiques, même dans les contextes non vernaculaires, entretient l'attachement à la langue, même s'il demeure en décalage avec les compétences réelles. Cela n'est pas surprenant : entre le désir de langue et la maîtrise d'une langue, il y a un chemin considérable, qui exige, hors contexte vernaculaire, des années d'investissement, ce dont peu d'acteurs du milieu sont réellement conscients.

\section{H\&M : Combien recense-t-on de locuteurs en} arménien en France ? La langue arménienne y est-elle restée vivante et parlée ? Quel impact a sa reconnaissance comme langue de France ?

A. D. : II n'y a aucun moyen aujourd'hui en France de recenser la communauté se reconnaissant d'origine arménienne, les chiffres évoqués, et qui tournent autour de 400000 à 500 000, sont des estimations faites à partir de recoupements. A fortiori, il est impossible d'inventorier les arménophones, sachant qu'il faudrait également déterminer le seuil à partir duquel on peut être considéré comme arménophone, ou encore savoir s'il est question de compétence passive (beaucoup plus répandue) ou active. Dans l'en- 


\section{REPÉRAGE}

quête en ligne que nous avons réalisée en 2014, et à laquelle ont répondu près de 1400 personnes, $85 \%$ des participants déclarent savoir lire et/ou écrire l'arménien, $80 \%$ déclarent savoir le lire et l'écrire, mais seuls $10 \%$ ont choisi le questionnaire en langue arménienne, et environ $7 \%$ ont utilisé l'arménien dans leurs réponses. La recon-

Aujourd'hui, environ 1300 élèves sont scolarisés en France dans les 7 écoles bilingues franco-arméniennes situées en Île-de-France, RhôneAlpes et Provence-Côte d'Azur. naissance de l'arménien occidental comme langue de France, même si elle est largement symbolique la France, qui reste un pays monolingue, n'a jamais ratifié la charte européenne des langues mino-

ritaires et régionales -, est un élément très précieux dans le mécanisme de maintien linguistique, tout comme la possibilité de présenter l'arménien au baccalauréat, introduite en 1984, mais malheureusement largement restreinte depuis. Aujourd'hui, environ 1300 élèves sont scolarisés en France dans les 7 écoles bilingues francoarméniennes situées en Île-de-France, RhôneAlpes et Provence-Côte d'Azur. En extrapolant le ratio combiné d'enfants scolarisés dans les cycles élémentaire et secondaire en France fourni par le ministère de l'Éducation nationale $(18,5 \%$ de la population), nous pouvons considérer que l'ensemble des élèves apprenant actuellement l'arménien dans les écoles bilingues et dans les cours hebdomadaires représente une population globale d'environ 15000 personnes. Ce chiffre ne prend cependant pas en compte la transmission familiale sans recours aux structures éducatives, ni les personnes ayant été scolarisées dans le passé en France ou à l'étranger et leurs descendants, ni les personnes ayant suivi des cours hebdomadaires. On peut avancer (avec prudence) que sur le chiffre de la population d'origine arménienne en France, habituellement estimée à 400 000, 25 à $35 \%$ pourraient être concernés par la langue arménienne à des degrés divers.
H\&M : Dans quels lieux peut-on apprendre aujourd'hui l'arménien en France ? L'école publique joue-t-elle son rôle dans l'apprentissage de cette langue ?

A. D. : Les 7 écoles bilingues franco-arméniennes sont des établissements privés, la plupart sous contrat avec l'État, qui délivrent le programme officiel français en y ajoutant l'arménien, dans des cours de langues des cours d'éveil ou des activités périscolaires. Elles scolarisent environ 1300 élèves. Les cours hebdomadaires sont également nombreux dans les structures associatives, culturelles ou religieuses, avec un maillage important dans toute la France, et concernent vraisemblablement environ le même nombre d'élèves. Le lycée Thiers à Marseille a inclus l'arménien parmi les langues facultatives, mais cela reste encore marginal. Actuellement, dans le prolongement des écoles existantes, la construction d'un lycée est en cours au Raincy, et un collège ouvre cette année à Alfortville, ce qui semble traduire plutôt une demande pour le privé.

\section{H\&M : Quels sont les profils et les motivations des étudiants qui décident d'apprendre l'armé- nien à l'Institut national des langues et civili- sations orientales (Inalco) ?}

A. D. : Les étudiants de l'Inalco représentent des profils très variés : certains ont déjà une bonne connaissance de l'arménien et effectuent un double cursus, soit en sciences humaines, soit dans une optique professionnelle, d'autres sont des doctorants qui ont rencontré un terrain arménien et souhaitent apprendre la langue. Enfin, certains combinent cette compétence linguistique avec d'autres langues de la région pour des carrières dans le commerce international ou les relations internationales. Nous jouons également un rôle important dans la formation des enseignants d'arménien des écoles, en formation continue ou initiale. Certains de nos anciens étudiants sont traducteurs indépendants auprès 
des tribunaux ou dans des structures d'accueil de migrants, d'autres travaillent dans le réseau associatif, d'autres encore dans I'humanitaire. Nous avons également un certain nombre d'étudiants de tous âges qui viennent à l'Inalco pour approfondir leurs connaissances de la langue qu'ils ont entendue étant enfants et qu'ils maîtrisent à des degrés divers.

H\&M : Les linguistes spécialistes de l'arménien dans le monde travaillent-ils en réseau pour comprendre les évolutions de ces langues en diaspora et leur relation actuelle avec celles pratiquées en Arménie?

A. D. : La linguistique arménienne est, comme la linguistique en général, un champ disciplinaire varié qui recouvre la grammaire comparée des langues indo-européennes anciennes, la philologie classique et la patristique, I'histoire de la langue, l'étude de sa structure phonétique, grammaticale, lexicale, l'étude de ses variations dialectales ou sociales, l'étude de son apprentissage ou de son acquisition. Des colloques réunissent régulièrement les linguistes du monde entier qui travaillent dans ces domaines et échangent leurs acquis. En Arménie également, depuis la chute de I'Union soviétique, des sections d'arménien occidental se sont ouvertes à l'université et à l'Académie des sciences d'Arménie. Plusieurs travaux ont décrit l'arménien occidental comme une langue pluricentrique et documenté ses différents états dans le monde. Cependant, beaucoup de linguistes s'attachent à répondre au recul linguistique par une approche normative, et les études de corpus permettant de documenter la langue parlée spontanée ne sont pas assez nombreuses pour pouvoir avoir une image réelle de la situation. Cela s'explique, d'une part, par le manque de prestige de la langue parlée par rapport à la langue écrite normée et standardisée et, d'autre part, par le fait que les études de corpus oraux demandent un investissement considérable en

termes de transcription et d'analyse. De plus, les chercheurs qui se consacrent à ces questions restent peu nombreux.

H\&M : Quelle est l'importance des médias en langue arménienne en France ? Certaines radios, des revues ou des journaux, des sites Internet, peuvent-ils être moteurs dans le maintien de cette langue?

A. D. : Il est essentiel. Sachant que très peu de Français d'origine arménienne lisent la littérature arménienne - Krikor Beledian, un des plus grands écrivains contemporains en arménien occidental, vit en France, mais n'est accessible qu'à une certaine élite, ce qui explique qu'il commence à être traduit en français -, les médias sont souvent la seule source extra-familiale d'arménien. Les trois grandes régions, Île-de-France, Rhône-Alpes et Provence-Côte d'Azur ont toutes leur radio arménienne, qui diffuse en deux langues, français et arménien, généralement occidental, mais sans exclure l'arménien oriental. La presse écrite arménienne en France peut se targuer d'avoir abrité le quotidien ayant eu la plus grande longévité en diaspora, le journal Haratch (En avant), fondé par Chavarche Missakian en 1925, et qui a paru jusqu'en 2009 sous une formule presque inchangée (4 pages, totalement en arménien, à l'exception de certaines annonces et publicités

L'édition numérique est une chance pour des langues comme l'arménien occidental, dont le public est dispersé dans le monde, ce qui complique le défi de la diffusion, chaque communauté n'étant pas une cible suffisante pour une politique éditoriale spécifique. en page 4) sous la direction de sa fille, Arpik Missakian, qui a résisté à la numérisation. À sa disparition, le trihebdomadaire Nor Haratch (Nouveau Haratch) a été créé par une nouvelle équipe, avec une version papier et une version numérique. Plusieurs mensuels sont aussi disponibles en langue française, certains (comme France-Arménie) ayant quelques pages 


\section{REPÉRAGE}

en arménien, dont le nombre tend à augmenter actuellement. Les contenus disponibles en arménien occidental sur Internet ont également beaucoup évolué. Plusieurs titres en arménien édités au Liban ou en Californie sont disponibles, les journaux télévisés en arménien de deux télévisions libanaises sont visibles sur YouTube quotidiennement, ce que peu savent en France. L'accès aux contenus en arménien oriental, notamment les chaînes de télévision incluses dans les bouquets câblés, joue un grand rôle dans la maîtrise de la langue. En effet, quelle que soit l'approche sur le futur de l'arménien occidental par rapport à l'arménien oriental, il est clair qu'un locuteur qui a accès à plusieurs variantes de la langue aura une meilleure compétence linguistique dans sa propre variante. L'édition numérique est une chance pour des langues comme l'arménien occidental, dont le public est dispersé dans le monde, ce qui complique le défi de la diffusion, chaque communauté n'étant pas une cible suffisante pour une politique éditoriale spécifique. De nombreux outils de référence sont actuellement disponibles sous forme numérique, notamment les dictionnaires, avec le site nayiri. com. C'est aussi le choix que nous avons fait en préparant un dictionnaire thématique françaisarménien occidental qui sera totalement gratuit en ligne.

\section{Bibliographie}

- Anaïd Donabédian, "Langues de diaspora, langues en danger : le cas de l'arménien occidental", in Mémoires de la Société de linguistique de Paris, nouvelle série, t. VIII, Les Langues en danger, 2000, pp. 137-156.

- Anaïd Donabédian (dir.), Langues de diaspora, langues en contact, faits de langue, Paris, Ophrys, $n^{\circ} 18,2001$.

- Anaïd Donabédian, "L'Arménien occidental", in Georg Kremnitz (dir.), Histoire sociale des langues de France, Rennes, PUR, 2013, pp. 609-618.

- Anaïd Donabédian, Anke Al-Bataineh, "L’arménien occidental en France, dynamiques actuelles", rapport remis à la DGLFLF, ministère de la Culture, octobre 2014. Accessible en ligne.

- Anaïd Donabédian, Sossé Manakian, Seta Bibérian, "Le défi des écoles bilingues franco-arméniennes", in Christine Helot, Jürgen Erfurt, L'Éducation bilingue en France. Politiques linguistiques, modèles et pratiques, Berne Peter Lang, 2015 [à paraître]. 